\title{
Dendrimer-based bionanomaterials produced by surface modification, assembly and hybrid formation
}

\author{
Kenji Kono \\ Because of their highly defined chemical structure and globular shape, dendrimers are very attractive as base materials to \\ be used in the production of nanomaterials for applications in various fields. Therefore, many attempts have been made \\ to develop functional materials using dendrimers. This review specifically examines dendrimer-based nanomaterials intended \\ for application to the biomedical field. The design, preparation and function of bionanomaterials, using various strategies \\ such as surface modification, assembly and hybrid formation, are described briefly along with their potential applications. \\ Polymer Journal (2012) 44, 531-540; doi:10.1038/pj.2012.39; published online 18 April 2012
}

Keywords: dendrimer; drug delivery system; hybrid; nanomaterial; self-assembly; stimuli-responsive; temperature-sensitive

\section{INTRODUCTION}

Dendrimers are a family of synthetic polymers with a regularly branched tree-like structure. ${ }^{1-4}$ Such a highly branched backbone gives dendrimers a globular shape that provides both a surface with certain properties and an interior that is capable of encapsulating guest materials. Furthermore, because molecular chains of dendrimers are grown stepwise by repeatedly introducing branch structures to their chain ends, their molecular size and structure can be controlled precisely. Uniformity at the molecular level and a high controllability of the molecular structure and properties make dendrimers highly attractive nanomaterials for application to various fields, including biomedical fields. ${ }^{5-8}$ It is necessary to give various functions to dendrimers to increase their usefulness considering their applications. One efficient approach to the functionalization of dendrimers is the attachment of functional groups to the chain ends of dendrimers. Dendrimer molecules have many chain terminals, which are located on their periphery. Therefore, if functional moieties are incorporated into these chain terminals, then crowding of these moieties can occur on the dendrimer surface, which might result in the amplification of these functions and/or the creation of new functions. Another efficient method to impart functions to dendrimers is hybrid formation with functional materials. The ability of dendrimers to encapsulate various guest materials enables complex formation with various functions, producing functional dendrimer-based hybrids. In addition, the defined molecular shape of dendrimers is beneficial to the components of the assemblies of highly ordered structures. Dendrimer-based assemblies may lead to the creation of new types of molecular assemblies with controlled morphologies. In this review, our efforts to develop functional dendrimer-based nanomaterials for biomedical applications, such as drug delivery, are described according to surface modification, hybrid formation and assembly strategies.

\section{POLY(ETHYLENE GLYCOL)-MODIFIED DENDRIMERS AS NANOCONTAINERS WITH BIOCOMPATIBLE SURFACES}

In terms of their use as biomaterials, dendrimers must have high biocompatibility. The toxicity of dendrimers is known to be affected strongly by their terminal groups. For example, poly(amidoamine) (PAMAM) dendrimers with primary amine terminal groups are toxic to various cells. However, dendrimers having the same backbone but different terminal groups, such as hydroxyl and carboxyl groups, exhibit much lower cytotoxicity. ${ }^{9}$ These findings suggest that the terminal groups of dendrimers have a central role in control of their cytotoxicity, most likely because the dendrimer backbone is covered by terminal groups. It is well established that surface modification with poly(ethylene glycol) (PEG) can provide excellent biocompatibility to nanoparticles (NPs) of various types, including polymeric micelles and liposomes. ${ }^{10}$ In particular, the high colloidal stability and long-term circulating properties of PEG-modified NPs in the bloodstream are of importance in terms of their use for targeted drug delivery because NPs with these properties can accumulate in target tumor tissues through so-called enhanced permeability and retention effects. Therefore, surface modification with PEG has been employed for dendrimers of various types to allow for their biological use. We have shown that PEG chains can be attached to all chain ends of PAMAM G3 and G4 dendrimers by the reaction of amine-terminated dendrimers and methoxy-PEG-4-nitrophenyl carbonate (Figure 1). ${ }^{11}$

Dendrimers of various types are shown to be capable of encapsulating small guest molecules. Indeed, PAMAM dendrimers can encapsulate various molecules. However, their encapsulation capability 

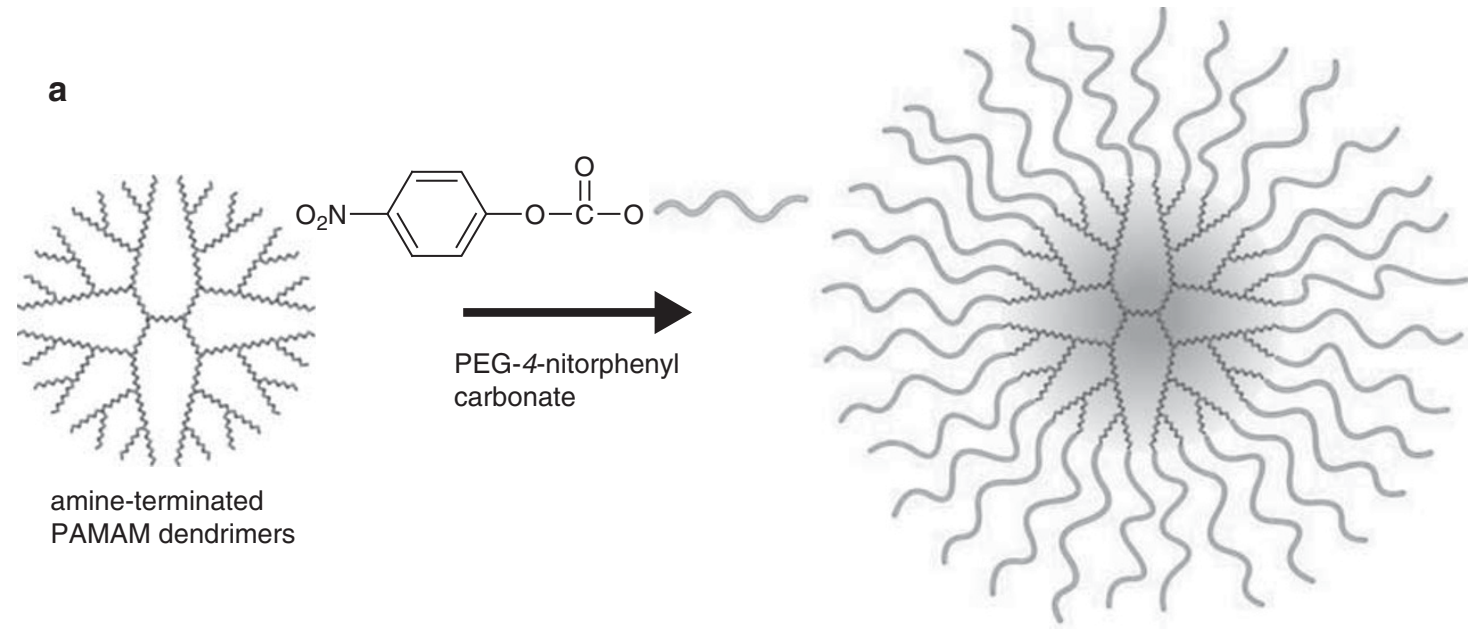

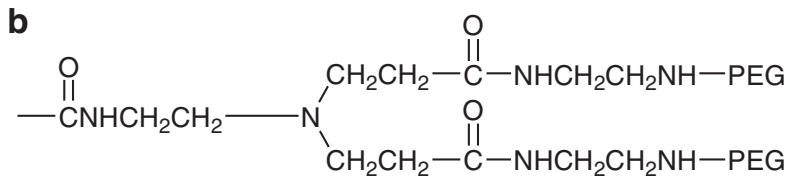

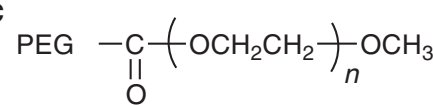

Figure 1 (a) Synthesis of poly(ethylene glycol) (PEG)-modified poly(amidoamine) dendrimers. Chemical structures of terminal moiety (b) and PEG chain (c) A full color version of this figure is available at Polymer Journal online.

varies depending on the chemical structure of guest molecules. In general, PAMAM dendrimers exhibit high encapsulation abilities toward molecules that have a hydrophobic backbone and negatively charged groups, such as benzoic acid ${ }^{12}$ and rose bengal, ${ }^{13}$ which can interact strongly with the PAMAM backbone through hydrophobic interaction and electrostatic interaction. We examined the effects of dendrimer generation and PEG chain length on the encapsulation ability of PEG-modified dendrimers for typical antitumor drugs, such as methotrexate and doxorubicin. As shown in Table 1, the dendrimers of higher generation with longer PEG chains exhibited higher encapsulation ability for both drugs, most likely because of the space created by their larger dendrimer interior and the larger hydration layers formed by PEG chains. ${ }^{11}$ In addition, PEG-modified PAMAM dendrimers exhibited higher encapsulation ability toward methotrexate, which has two carboxyl groups in the molecule, compared with doxorubicin. Their drug-release behaviors were also examined. When dialyzed against saline, the methotrexate-loaded PEG-modified PAMAM dendrimers released the drug quickly, indicating that their capability of retaining drug molecules might not be sufficient to deliver drugs to their target tissue efficiently.

\section{PEG-MODIFIED PAMAM DENDRIMERS WITH FUNCTIONAL SHELLS}

Several strategies can be used to improve the performance of dendrimers as nanocontainers. One possible approach is to modify the dendrimer backbone structure to increase its affinity to drug molecules. Indeed, the quaternization or hydrophobic modification of the dendrimer backbone is possible. In fact, the increase of hydrophobicity in the dendrimer backbone was shown to improve the encapsulation ability of dendrimers greatly. ${ }^{14}$ Another approach is the construction of a shell onto the dendrimer surface, suppressing or
Table 1 Encapsulation of anticancer drugs by PEG-modified poly(amidoamine) dendrimers

\begin{tabular}{lcc} 
Dendrimer & Maximum number of encapsulated molecules per dendrimer \\
\hline & & \\
PEG(550)-G3 & Doxorubicin & methotrexate \\
PEG(2000)-G3 & 1.2 & 10.3 \\
PEG(550)-G4 & 2.4 & 13.3 \\
PEG(2000)-G4 & 1.8 & 19.7 \\
& 6.6 & 26.4 \\
\hline
\end{tabular}

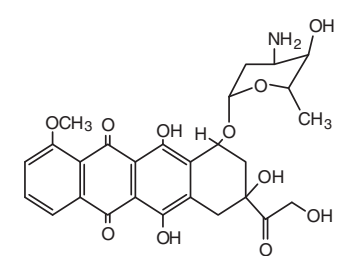<smiles>CN(Cc1cnc2nc(N)nc(N)c2n1)c1ccc(C(=O)NC(CCC(=O)O)C(=O)O)cc1</smiles>

Abbreviation: PEG, poly(ethylene glycol).

controlling the diffusion of the drug molecules from the dendrimer interior to the outside of the dendrimer. For example, attachment of protected amino acids onto poly(propyleneimine) (PPI) dendrimers generates a densely packed shell that locks guest molecules in the dendrimer interior. ${ }^{15}$

Considering previously reported results, we incorporated shell structures consisting of hydrophobic amino acids, such as phenylalanine (Phe) and glutamic acid- $\gamma$-benzyl ester $(\mathrm{Glu}(\mathrm{OBzl}))$, onto the surface of PAMAM dendrimer moiety (Figure 2a,b). ${ }^{16}$ The molecular weights and diameters of PEG-modified PAMAM G4 dendrimers 
a

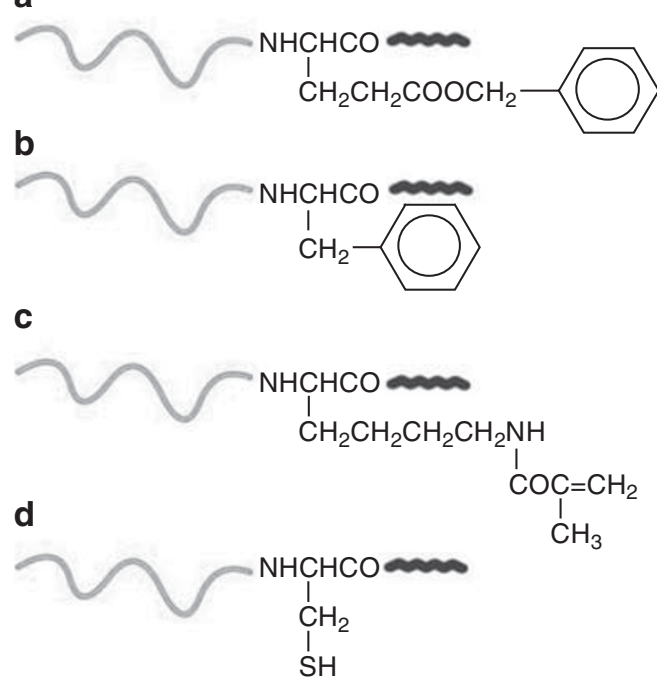

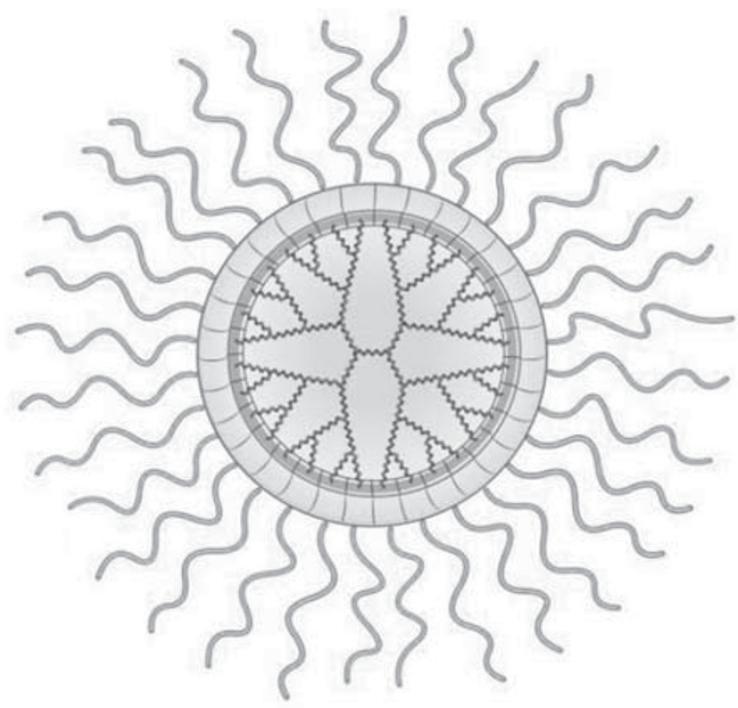

Figure 2 Design of poly(ethylene glycol) (PEG)-modified poly(amidoamine) dendrimers with functional shells. PEG-modified dendrimers with Glu(OBzl) (a) Phe (b), Lys( $\epsilon$-methacrylamide) (c), and Cys (d) residues. A full color version of this figure is available at Polymer Journal online.

Table 2 Molecular weights and hydrodynamic diameters of PEG-modified dendrimers

\begin{tabular}{|c|c|c|c|c|c|c|}
\hline \multirow[b]{3}{*}{ Dendrimer } & \multicolumn{4}{|c|}{ Molecular weight } & \multicolumn{2}{|c|}{ Diameter \pm s.d. $(\mathrm{nm})$} \\
\hline & \multirow[b]{2}{*}{ Calculated } & \multicolumn{3}{|c|}{ Estimated by GPC } & \multicolumn{2}{|c|}{ Estimated by $D L S$} \\
\hline & & $M n$ & $M w$ & $\begin{array}{l}M w / \\
M n\end{array}$ & $\begin{array}{l}\text { Number } \\
\text { average }\end{array}$ & $\begin{array}{l}\text { Weight } \\
\text { average }\end{array}$ \\
\hline PEG-G4 & 143879 & 35300 & 46400 & 1.3 & $15.3 \pm 1.1$ & $16.2 \pm 2.2$ \\
\hline PEG-Phe-G4 & 153297 & 29300 & 36200 & 1.2 & $14.2 \pm 2.5$ & $15.5 \pm 2.0$ \\
\hline $\begin{array}{l}\text { PEG- } \\
\text { Glu(OBzl)-G4 }\end{array}$ & 157910 & 26900 & 40200 & 1.5 & $12.0 \pm 1.8$ & $13.0 \pm 2.1$ \\
\hline
\end{tabular}

Glu(OBzl)-G4

Abbreviations: DLS, dynamic light scattering; GPC, gel permeation chromatography; PEG, poly(ethylene glycol).

Molecular weights of dendrimers were calculated assuming that molecular weight of PEG is 2000. Phosphate $(10 \mathrm{~mm})$ and $0.2 \mathrm{M} \mathrm{Na}_{2} \mathrm{SO}_{4}$ solution $(\mathrm{pH} 7.4)$ was used as eluent.

Poly(ethylene glycol)s were used as standards

with the shell of Phe or Glu(OBzl) residues, as well as those for the parent dendrimer without amino-acid residues, are presented in Table 2. Theoretically, dendrimers having amino-acid residues can be expected to have higher molecular weights than PEG-modified dendrimers without amino-acid residues. However, these aminoacid-incorporated PEG-modified dendrimers were smaller in size, which indicates that amino-acid residues attached to the dendrimer periphery moiety interact through hydrophobic interaction and form a shell, making the dendrimer chains more compact.

The shell consisting of hydrophobic amino-acid residues improved the performance of the PEG-modified dendrimer as a nanocontainer. Table 3 shows the binding constant and the number of binding sites of the PEG-modified PAMAM G4 dendrimers with and without the amino-acid shells for rose bengal. These dendrimers exhibited much larger binding constant values, and the number of binding sites at a $\mathrm{pH}$ of 5.0 is much greater than that at a $\mathrm{pH}$ of 7.4 because protonation of tertiary amines in the dendrimer interior increased the capability of the dendrimers to bind to negatively charged rose bengal molecules. The comparison of the binding capability among these
Table 3 Binding constants and numbers of binding sites of dendrimers for rose bengal

\begin{tabular}{|c|c|c|c|c|c|c|}
\hline \multirow[b]{2}{*}{ Dendrimer } & \multicolumn{3}{|c|}{ pH 7.4} & \multicolumn{3}{|c|}{ pH 5.0} \\
\hline & $n k\left(\mathrm{M}^{-1}\right)$ & $\mathrm{n}$ & $k$ & $n k\left(\mathrm{M}^{-1}\right)$ & $n$ & k \\
\hline PEG-G4 & $2.0 \times 10^{6}$ & 9 & $2.2 \times 10^{5}$ & $1.8 \times 10^{8}$ & 43 & $4.2 \times 10^{6}$ \\
\hline PEG-Phe-G4 & $2.1 \times 10^{7}$ & 11 & $1.9 \times 10^{6}$ & $2.8 \times 10^{8}$ & 37 & $7.6 \times 10^{6}$ \\
\hline PEG-Glu(OBzI)-G4 & $3.1 \times 10^{7}$ & 12 & $2.6 \times 10^{6}$ & $3.3 \times 10^{8}$ & 44 & $7.5 \times 10^{6}$ \\
\hline
\end{tabular}

Abbreviation: PEG, poly(ethylene glycol).

dendrimers at the same $\mathrm{pH}$ shows that the binding constant greatly increased for the dendrimers with the shell, although these dendrimers showed similar binding site quantities, suggesting that the shell can cause the guest molecules to be more tightly bound to the dendrimer chain. In addition, the hydrophobic amino-acid shell can effectively suppress the release of rose bengal from the dendrimer interior at a $\mathrm{pH}$ of 3, where the PEG-modified dendrimer without a shell released the guest molecules quickly. ${ }^{16}$

Another approach to the construction of a shell on the dendrimer periphery is through the linking of the polymerizable groups on the dendrimer surface. Zimmerman et al. ${ }^{17}$ demonstrated that the ringclosing polymerization of vinyl groups attached to the chain terminals of poly(arylether) dendrimers can be induced within a single dendrimer molecule when polymerization is performed under dendrimer-diluted conditions, ${ }^{18,19}$ and they used this method to create a shell on the dendrimer surface to achieve monomolecular imprinting inside dendrimers. ${ }^{17}$ We also attempted to produce a shell by linking methacryloyl groups attached to the periphery of the dendrimer moiety of the PEG-modified PAMAM G4 dendrimer. Methacryloyl groups were combined to side chains of L-lysine residues of the L-lysine shell-incorporated PEG-modified dendrimer (Figure 2c). These polymerizable groups were actually linked within a single dendrimer molecule using free radical polymerization because intermolecular polymerization may be prohibited sterically by PEG chains grafted to the dendrimer surface. ${ }^{20}$ When the polymerization 
was performed for the same dendrimer encapsulating 10 rose bengals per dendrimer, the obtained dendrimer retained the guest molecule tightly. Although the number of the rose bengal entrapped in a dendrimer was 0.4 per dendrimer, the result suggests that the linking of chain terminal of dendrimers might be applied to physically lock guest molecules inside the dendrimer.

When the dendrimers are combined with a shell whose formation and dissociation are controlled in response to stimuli irradiation or environmental change, the resultant dendrimers are expected to exhibit stimulus-responsive or environment-responsive properties. Based on this idea, we attached L-cysteine residues onto the dendrimer surface moiety of PEG-modified PAMAM G4 dendrimer (Figure 2d). ${ }^{21}$ A thiol group of a cysteine side group can form a disulfide linkage with the thiol group of an adjacent cysteine residue in an oxidative environment, resulting in the formation of a dense shell. However, in a reductive environment, the disulfide linkage is cleaved to generate thiol groups. In fact, PEG-modified dendrimers that have a shell of linked cysteine residues exhibited a more than 10 -fold greater binding constant in the presence of the reductive reagent dithiothreitol compared with the oxidative environment in the absence of dithiothreitol. These findings indicate that the introduction of a shell onto the dendrimer surface is an efficient approach to the production of nanocontainers with various functions.

\section{TEMPERATURE-SENSITIVE DENDRIMERS BY SURFACE MODIFICATION}

Polymers with stimulus-sensitive properties have been used widely for the production of functional materials with applications in various fields, including biomedical fields. For example, stimulus-sensitive NPs are studied intensively for their use as drug delivery systems because their functions can be controlled by the application of stimuli from outside the body. Indeed, if stimulus-sensitive properties are provided to dendrimers, then the range of their application fields is expected to be expanded greatly, and their usefulness as functional nanomaterials is also expected to increase dramatically. From the viewpoint of stimulus-sensitivity, several studies have been conducted using dendrimers. Indeed, widely used PAMAM dendrimers and PPI dendrimers have $\mathrm{pH}$-sensitive tertiary amine groups in their respective backbones. Therefore, these dendrimers behave as $\mathrm{pH}$-sensitive nanocontainers. ${ }^{22-24}$ In addition, photosensitive moieties, such as photoisomerizable azobenzene $e^{25}$ and photocleavable o-nitrobenzene ${ }^{26}$ residues, were attached to the chain terminal of PPI or PAMAM dendrimers to provide photosensitive dye molecule encapsulation or drug-release properties to these dendrimers.

Temperature is a useful, safe and readily applicable stimulus from a practical viewpoint. Therefore, many attempts have been made to provide thermosensitive properties to various NPs, such as polymeric micelles $^{27,28}$ and vesicles. ${ }^{29-31}$ These thermosensitive systems have usually been prepared using thermosensitive polymers, which change the water solubility depending on temperature, ${ }^{32}$ and one particular example is $\operatorname{poly}(N$-isopropylacrylamide) [poly(NIPAAm)], which exhibits a lower critical solution temperature (LCST) at ca. $32{ }^{\circ} \mathrm{C} .{ }^{33}$ This polymer is highly soluble in water at a low temperature, but it becomes water insoluble above the LCST. In addition, the LCST of such thermosensitive polymers can be changed by copolymerization with a comonomer of appropriate hydrophilicity or hydrophobicity. ${ }^{34,35}$

Thermosensitive polymers have also been used to provide temperature-sensitive properties to dendrimers. Dendrimers of various types were combined with thermosensitive polymers using various synthetic methods. For example, poly(NIPAAm)-grafted PPI dendrimers were prepared using free radical polymerization of
NIPAAm with a thiol-terminated dendrimer as a chain transfer reagent. ${ }^{36}$ Reversible addition-fragmentation chain transfer (RAFT) polymerization of NIPAAm using dithiobenzoyl group-terminated dendrimers as a RAFT reagent was also utilized for the preparation of dendrimers having poly(NIPAAm) chains with controlled chain length. ${ }^{37}$ Similarly, thermosensitivepoly $(\mathrm{N}, \mathrm{N}$-dimethylaminoethyl methacrylate) chains were grafted onto a PAMAM dendrimer via atom transfer radical polymerization. ${ }^{38}$ In addition, poly(NIPAAm) with 4-nitrophenyl-carbonate-activated terminal was reacted with amine-terminated PAMAM dendrimers to obtain temperaturesensitive dendrimers. ${ }^{39}$ These thermosensitive polymer-attached dendrimers exhibit temperature-controlled functions such as particle size, ${ }^{37}$ catalytic activity ${ }^{36}$ and drug-release behaviors. ${ }^{38,39}$ However, dendrimers of this type are expected to exhibit a lowering of their structural uniformity. Their molecular shape might be star-like rather than spherical. Their thermosensitive properties indeed originated from their thermosensitive polymer chains. Consequently, thermosensitive dendrimers of this type necessarily spoil these important features of dendrimers.

We have found another method to render dendrimers temperaturesensitive without disturbing their molecular uniformity: surface modification with thermosensitive groups (Figure 3). ${ }^{40}$ Because the interaction of the side groups of thermosensitive polymers has an important role in the generation of their thermosensitive properties, the attachment of these groups to the chain terminals of dendrimers is expected to induce close contact of these groups, generating the thermosensitive surface of the dendrimers. Because the isobutyramide (IBAM) group is the side group of poly( $N$-vinyl isobutyramide), which is a thermosensitive polymer with a LCST of ca. $39^{\circ} \mathrm{C}$, ${ }^{41}$ we incorporated the IBAM group into every chain end of the PAMAM dendrimers. As presented in Figure 4, the IBAM-terminated PAMAM G2 dendrimer was soluble in water in the experimental temperature region, but those of higher generations exhibited a cloud point at a specific temperature, which is indicative of the LCST. Additionally, it is apparent that the cloud point of these dendrimers decreased concomitantly with increasing dendrimer generation. The dendrimer with a higher generation should have a surface with a higher density of IBAM groups. Therefore, more efficient interaction between the IBAM groups might cause their dehydration at a lower temperature.

Surface modification with IBAM groups was applicable for temperature sensitization to dendrimers of other types. For example, IBAM groups were attached to all terminals of the PPI G4 and G5 dendrimers. These IBAM-terminated dendrimers also exhibited an LCST, but their LCST was lower by $30-40{ }^{\circ} \mathrm{C}$ than that of the PAMAM dendrimers with the same quantities of the terminal groups. ${ }^{40} \mathrm{PPI}$ dendrimers have a smaller molecular size than PAMAM dendrimers of the same number of chain terminals. Therefore, the density of IBAM groups in the dendrimer periphery is expected to be higher for IBAM-terminated PPI dendrimers than for the corresponding IBAMterminated PAMAM dendrimers, indicating that the interaction of the IBAM groups in the dendrimer periphery is responsible for the generation of temperature-sensitive properties.

The LCST of thermosensitive polymers with linear structure, such as poly( $N$-alkylacrylamide)s and poly $(N$-vinylalkylamide)s, is well known to be affected by the chemical structure of side groups, ${ }^{42,43}$ as is also the case for the thermosensitive dendrimers with globular structure. For example, PAMAM G4 dendrimers having $n$-butyramide groups, IBAM groups and cyclopropane-carbamide groups on the surface exhibited LCST at 45,61 and $15^{\circ} \mathrm{C},{ }^{44}$ respectively. When terminal groups of two types were attached to the PAMAM dendrimers, the resultant dendrimers showed LCST at different 


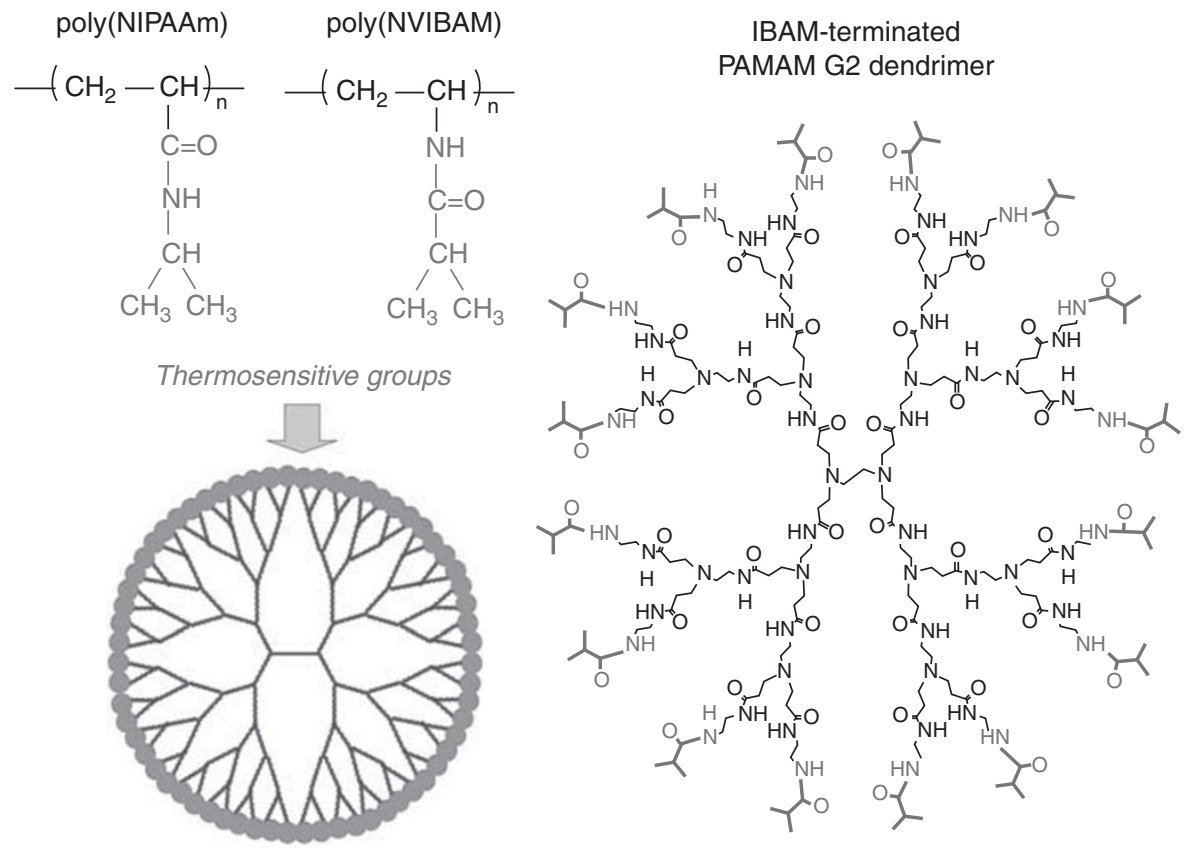

Figure 3 Design of thermosensitive dendrimers. Chemical structures of typical thermosensitive polymers, poly(N-isopropylacrylamide) [poly(NIPAAm)] and poly( $N$-vinyl isobutyramide) [poly(NVIBAM)], and isobutyramide (IBAM)-terminated poly(amidoamine) G2 dendrimer. A full color version of this figure is available at Polymer Journal online.

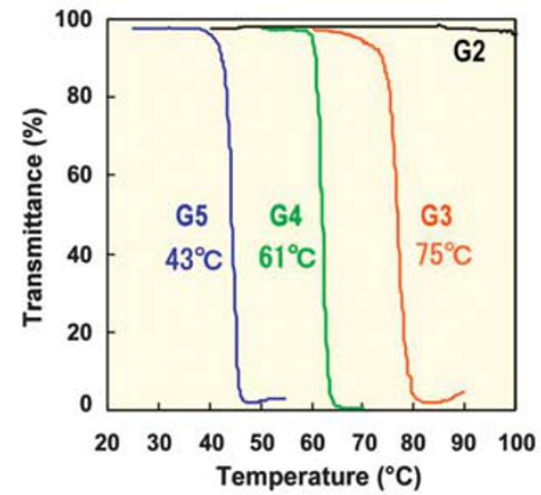

IBAM-G2

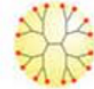

IBAM-G3

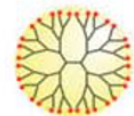

IBAM-G4

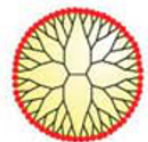

IBAM-G5

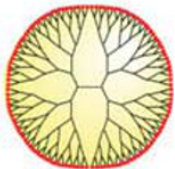

Figure 4 Effect of temperature on transmittance of isobutyramide (IBAM)terminated poly(amidoamine) dendrimers with varying generations $\left(10 \mathrm{mg} \mathrm{ml}^{-1}\right.$ ) dissolved in $10 \mathrm{~mm}$ phosphate solution ( $\mathrm{pH} 9.0$ ).

temperatures, depending on their terminal group composition, indicating that dendrimers that exhibit temperature sensitivity at a desired temperature are obtainable by controlling the composition of the terminal groups.

Fundamentally, it is of interest to understand how differently the globular shape of these thermosensitive dendrimers influences their thermosensitive property compared with that of the linear polymers. A significant difference was found for their endotherm at the LCST. ${ }^{45}$ Thermosensitive polymers such as poly(NIPAAm) are known to exhibit a large endotherm at the LCST, which is explained by the release of structured water around hydrophobic isopropyl groups upon structural transition from a hydrated coil to a dehydrated globule at the LSCT. ${ }^{33,34}$ We compared thermosensitive properties of

PAMAM dendrimers having $N$-isopropylamide (NIPAM) groups on all chain terminals with those of poly(NIPAAm) and NIPAAm copolymers, which revealed that both these polymers and dendrimers similarly exhibited a sharp water-solubility change at a specific temperature (Figure 5). ${ }^{45}$ However, NIPAM-terminated dendrimers exhibited much lower transition enthalpies at the LCST than did the NIPAM-bearing linear polymers. The NIPAM-bearing linear polymers' large conformational freedom enables the efficient hydration of the NIAPM groups below the LCST and the efficient association of the NIPAM groups above the LCST, whereas the NIPAM-terminated dendrimers with low conformational freedom might generate dense packing of the NIPAM groups in the periphery, which engenders insufficient hydration below the LCST and insufficient dehydration above the LCST. The surface modification of dendrimers with thermosensitive groups enables the generation of a new type of thermosensitive polymers that undergo a sharp transition by dehydration of the peripheral moiety without a large conformational change in the whole molecule. ${ }^{45}$ These thermosensitive dendrimers can change surface hydrophilicityhydrophobicity in response to temperatures by the retention of guest molecules in their interior. $^{46}$ Such a function might expand dendrimers' potential applications as smart nanomaterials and increase their usefulness.

To date, several types of thermosensitive dendrimers exhibiting LCST have been prepared through the surface modification of dendrimers with various groups. These include hydrophobic aminoacid residues, such as L-phenylalanine and L-leucine residues ${ }^{47}$ and carborane ${ }^{48}$ In addition, surface modification has been applied to provide temperature-sensitive properties to hyperbranched polymers of various backbone structures including polyamidoamines, ${ }^{49}$ poly(ethyleneimine $)^{50,51}$ and poly(glycidol)..$^{52-55}$ As another type of thermosensitive polymers, dendrimers and hyperbranched polymers consisting of thermosensitive polyether backbones have also been developed. ${ }^{56,57}$ 

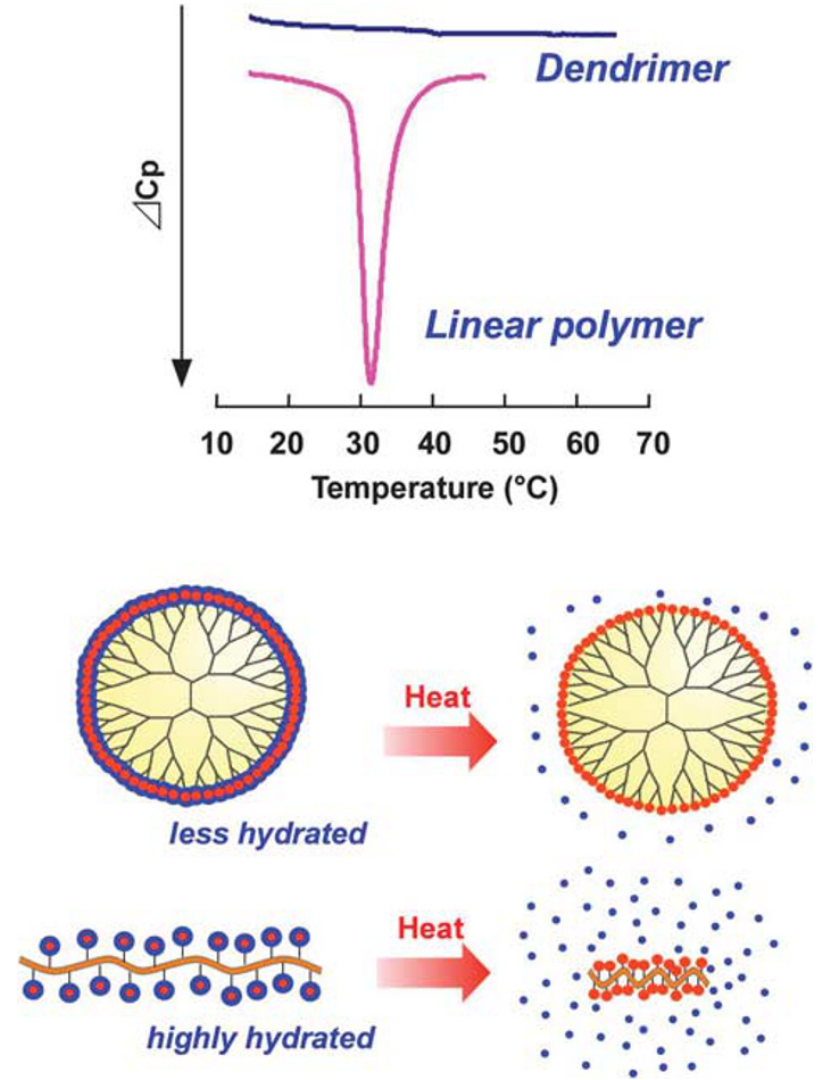

Figure 5 Comparison of transition between poly(NIPAAm) and $\mathrm{N}$-isopropylamide (NIPAM)-terminated dendrimer. DSC charts for poly(NIPAAm) and NIPAM-terminated poly(amidoamine) G5 dendrimer (Transition temperature: $40.4{ }^{\circ} \mathrm{C}$ ) and their transition mechanisms are shown.

\section{DENDRIMER-BASED AMPHIPHILES FOR FUNCTIONAL MOLECULAR ASSEMBLIES}

Molecular assemblies, such as vesicles and micelles, are widely used and important nanomaterials in biomedical applications, such as in drug delivery. These systems are formed by amphiphiles in aqueous media through their hydrophobic interaction. In general, the characteristics of the molecular assemblies, such as size, stability and morphologies, are affected by the chemical structures of the amphiphiles that are used as the components. ${ }^{58}$ Therefore, the design of the amphiphiles might be an efficient approach to the production of molecular assemblies with excellent performance. Dendrimer-based amphiphiles are attractive as components of molecular assemblies because of the large freedom in their molecular design from the perspectives of size control, molecular shape and physicochemical properties. ${ }^{59}$

Amphiphilic molecules of various types have been prepared for the formation of molecular assemblies in aqueous solutions. For example, PPI dendrimers terminally functionalized with aliphatic chains have been shown to form vesicles because these cylindrical amphiphilic dendrimers associate through hydrophobic interaction and form bilayer membranes. ${ }^{60,61}$ In addition, hybrid molecules consisting of hydrophilic dendron and hydrophobic linear polymers have been prepared. These amphiphilic macromolecules have been shown to form micelles and vesicles, depending on the balance between the dendron and linear polymer moieties. ${ }^{62,63}$
As a dendrimer-based amphiphile, we have developed a new type of lipid consisting of a head group of a PAMAM dendron and two tails of hydrophobic alkyl chains. ${ }^{64,65}$ The selections of the generation of a PAMAM dendron moiety and alkyl chain length afford dendronbearing lipids of varying head-to-tail balances. ${ }^{64,65}$ In addition, the surface modification of the dendron moiety can provide dendronbased lipids with various functional characteristics, such as multi-PEG chains for improved colloidal stability ${ }^{66}$ or sugar residues for targetspecific properties. ${ }^{66}$ These dendron-based lipids are useful as potent vectors for gene transfection because these molecules form complexes with plasmid DNA through electrostatic interaction and interact strongly with cells. In fact, these dendron-lipid-plasmid DNA complexes, which are taken up efficiently by various cells types, engender gene expression. ${ }^{64-67}$

When dendron-based lipids having functional groups on the dendron chain ends were used, these functional groups allowed for the formation of a highly concentrated surface by virtue of their assembly formation. According to this approach, thermosensitive vesicles of a new type are obtained using IBAM-terminated PAMAM dendron-lipids (Figure 6). ${ }^{68}$ In aqueous solutions, these IBAMterminated dendron-lipids form vesicles through the hydrophobic interaction of their long alkyl chains, generating a thermosensitive IBAM-concentrated surface on the vesicles. In fact, the IBAMterminated G2 and G3 dendron-lipids form vesicles with diameters of $50-100$ and $100-200 \mathrm{~nm}$, respectively, at low temperatures, but these vesicles become unstable and form aggregates at approximately 40 and $50^{\circ} \mathrm{C}$, respectively. It is particularly interesting that the morphology of the IBAM-terminated G2 dendron-lipid vesicles transformed into a rod-like shape after aggregation. Most likely, the dehydration of the dendron moiety induces a change in the dynamic molecular shape of the dendron lipid from cylindrical to truncated-conic, which are suitable for lamellar formation and inverted rod-like micelle formation, respectively ${ }^{58}$ In contrast, the IBAM-terminated G3 dendron-lipid vesicles retain vesicular shape aggregation, but their size increased, most likely because of the fusion of the vesicles.

Practically, it is important to control the temperature region in which the dendron-based lipid vesicles exhibit their response, and this can be achieved by mixing the dendron-bearing lipids with different thermosensitivities at an appropriate ratio for the vesicle preparation. For example, the cloud point of the vesicles consisting of the IBAMterminated dendron lipid and the ACAM (acetamide)-terminated dendron-lipids increased with increasing ACAM-terminated dendron-lipid content because the hydrophilic nature of the ACAM group enhances the hydration of the vesicle surface. Therefore, vesicles that exhibit the response at a desired temperature region are obtainable by adjusting the ratio of these dendron-lipids. In contrast to conventional thermosensitive vesicles, which exhibit a thermal response through a gel-to-liquid crystalline transition of their membrane, ${ }^{69}$ these dendron-lipid vesicles are a new type of thermosensitive vesicle whose destabilization is induced by the dehydration of the vesicle surface.

\section{DENDRIMER-GOLD NP HYBRIDS}

Hybrid formation with functional materials is another efficient method to impart functions to dendrimers. Using the ability of dendrimers to encapsulate various guest materials, complexes of various functions can be prepared. One attractive material for the functionalization of dendrimers via the complex formation strategy is metal NPs. In fact, several studies have investigated the production of dendrimer-encapsulated NPs of various metals 

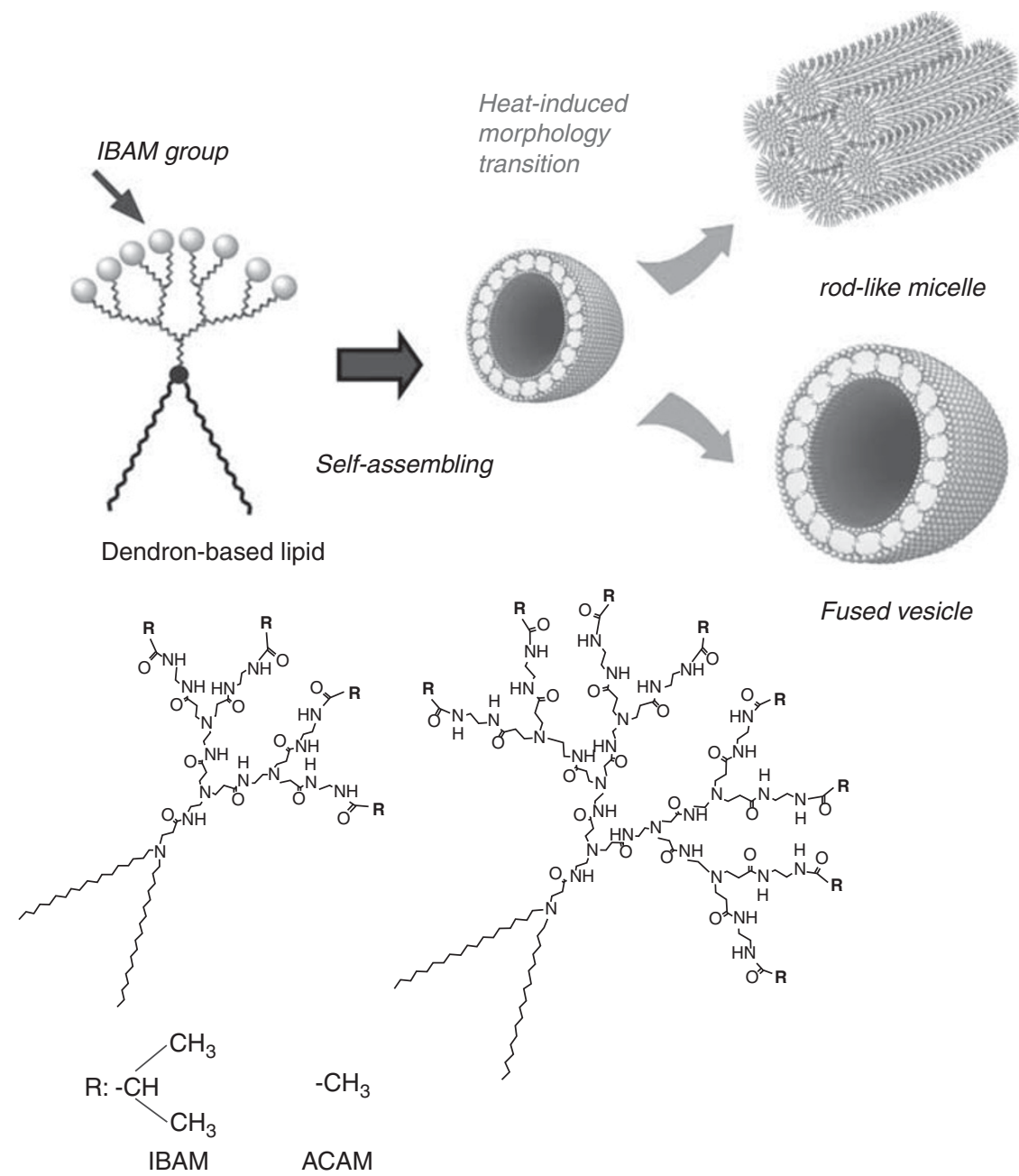

Figure 6 Design of thermosensitive molecular assemblies using dendron-based lipids. Chemical structures of isobutyramide (IBAM)-terminated and acetamide (ACAM)-terminated G2 and G3 dendron-lipids are shown. A full color version of this figure is available at Polymer Journal online.

such as $\mathrm{Au}, \mathrm{Cu}, \mathrm{Ag}, \mathrm{Pt}, \mathrm{Pd}$ and $\mathrm{Rh}$ for various applications, ${ }^{70,71}$ including those related to catalysis, ${ }^{72,73}$ optics, ${ }^{74}$ sensors, ${ }^{75}$ imaging $^{76-78}$ and biomedicine. ${ }^{79}$

For applications in the biomedical field, Au NPs are attractive because of their heat-generating property under visible and nearinfrared light irradiation, which is useful for the detection and treatment of tumors. ${ }^{80-82}$ This unique physical property of Au NPs is derived from the excitation of surface plasmons by light, designated as surface plasmon resonance and the subsequent conversion to heat. ${ }^{83}$ Therefore, the complexation of Au NPs with dendrimers can engender the generation of new nanomaterials with photothermal functions.

Uniform Au NPs with 2-4-nm diameters are obtainable by the loading of $\mathrm{HAuCl}_{4}$ in the PAMAM dendrimers of various generations and by subsequent reduction with an appropriate reducing agent such as $\mathrm{NaBH}_{4}{ }^{84}$ Considering their applications to bionanomaterials, PEG-modified PAMAM dendrimers have been used for complex formation with Au NPs. ${ }^{85-87}$ As is the case of unmodified PAMAM dendrimers, PEG-modified PAMAM dendrimers are useful as a template for the formation of $\mathrm{Au}$ NPs. When $\mathrm{AuCl}_{4}{ }^{-}$ions were reduced within the PEG-modified PAMAM G4 dendrimer, Au NPs with average diameters of approximately $2 \mathrm{~nm}$ were generated within the dendrimer. The Au NPs encapsulating PEG dendrimers were stable even after freeze drying, most likely because the steric PEG chains isolate Au NPs within the dendrimers and suppress their aggregation between the dendrimers (Figure 7). ${ }^{86}$ Additionally, it was possible to grow $\mathrm{Au}$ NPs in the dendrimer interior by repeating $\mathrm{AuCl}_{4}{ }^{-}$ion loading and subsequent reduction with $\mathrm{NaBH}_{4}$ in the dendrimer interior. The Au NP size was increased from 2 to $4-5 \mathrm{~nm}$, which is approximately equal to the size of a PAMAM G4 dendrimer, by repeating the Au loading process 10 times. The Au NPs encapsulated in the PEG-modified dendrimers can be activated by irradiation with visible light $(\lambda=532 \mathrm{~nm})$ and can generate heat effectively. Indeed, the heat-generating ability under light irradiation increases with the increasing size of the $\mathrm{Au}$ NPs, not only because of the increased amount of Au NPs in the dendrimer but also because of the more efficient surface plasmon resonance of the well-grown Au NPs. ${ }^{87}$ When cells were incubated with these PEG-modified dendrimer-Au NP hybrids, these hybrid particles are taken up by cells through endocytosis. After treatment with the hybrids, the cells were killed by light irradiation. In addition, the cells treated with the hybrids having larger Au NPs were shown to be killed more effectively under light irradiation, suggesting their possible application to photothermal therapy (Figure 8), and 


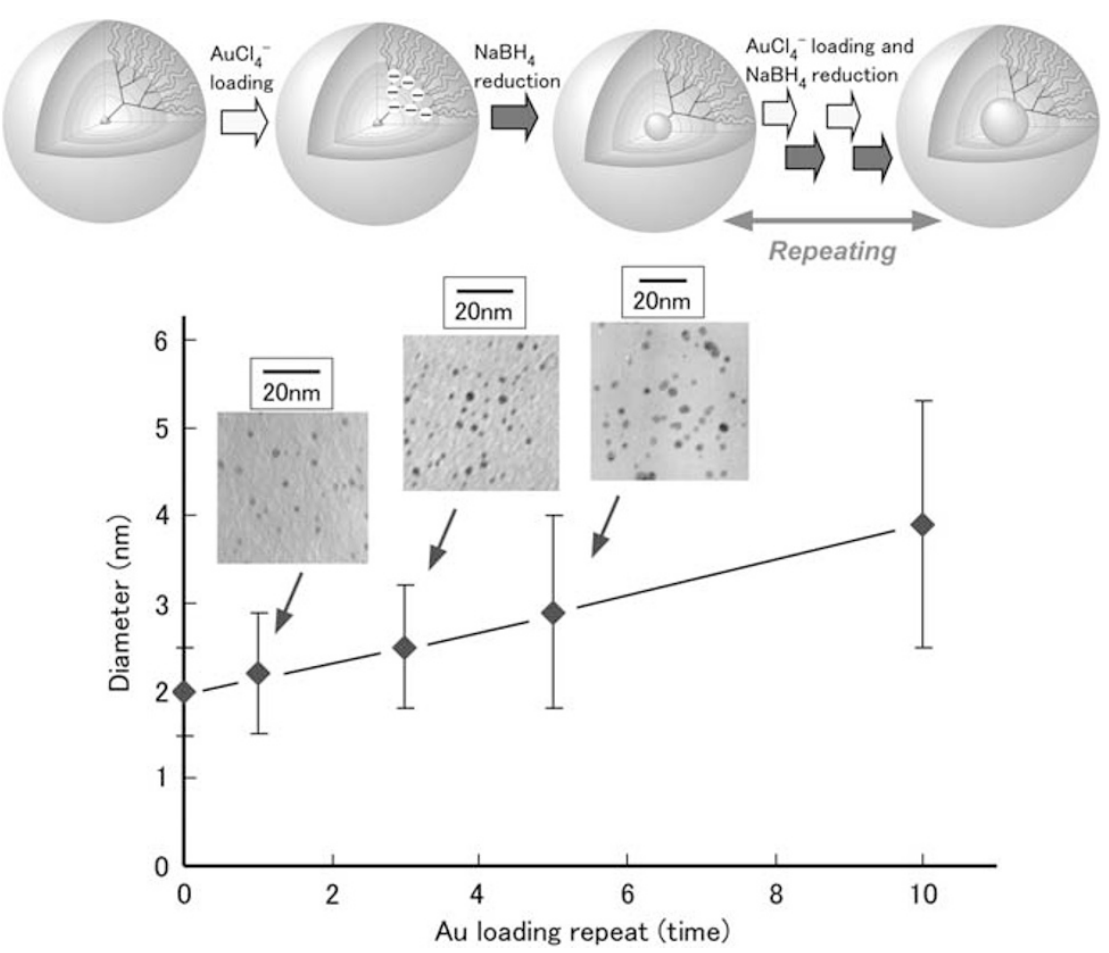

Figure 7 Preparation of Au nanoparticle (NP)-loaded poly(ethylene glycol)-modified poly(amidoamine) G4 dendrimers. Au NPs were grown in the dendrimers by repeating $\mathrm{AuCl}_{4}{ }^{-}$loading and its reduction. Mean diameter, s.d. and transmission electron microscope images of Au NPs formed in the dendrimers are shown. A full color version of this figure is available at Polymer Journal online.

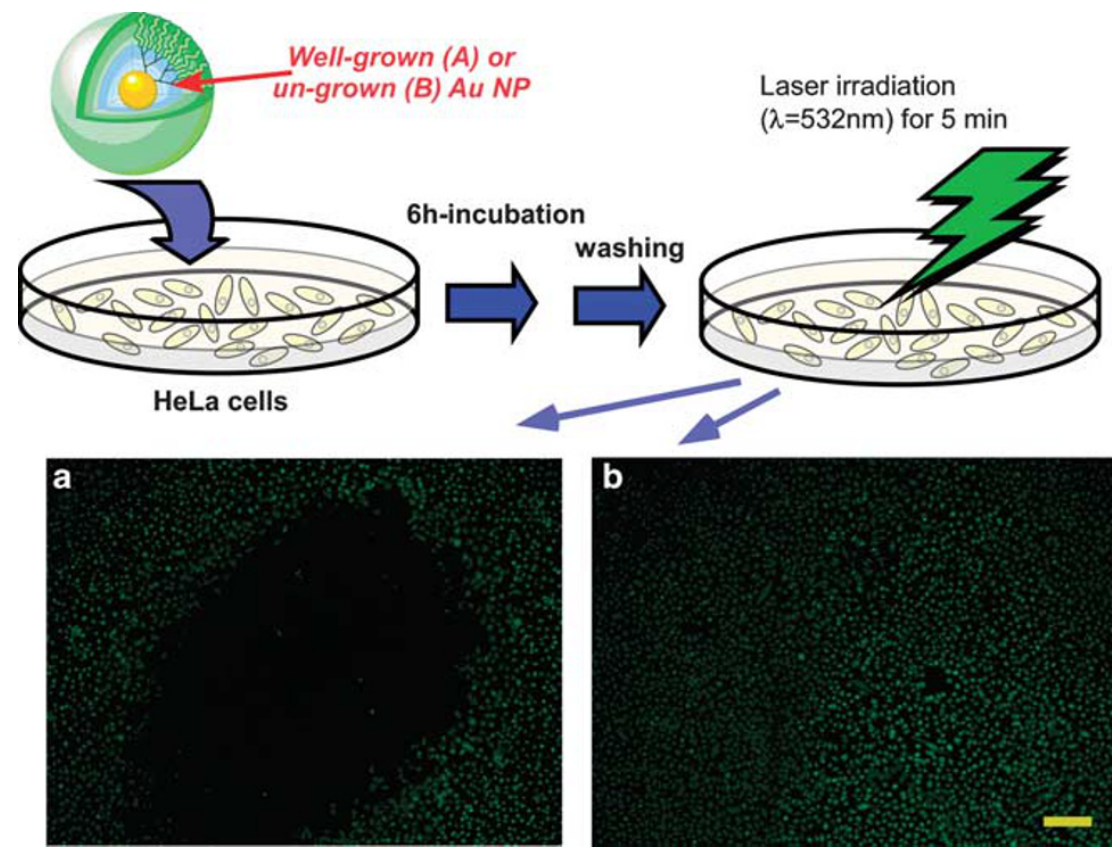

Figure 8 Photo-induced cytotoxicity of the poly(ethylene glycol)-modified poly(amidoamine) G4 dendrimers encapsulating well-grown Au NPs by repeating Au loading five times (a) or un-grown Au NPs (b). Alive cells were stained with calcein-AM. Fluorescence micrographs (a) and (b) show the regions around the photo-irradiated area. Scale bar indicates $200 \mu \mathrm{m}$.

considering this potential application, it is worth noting that the dendrimer-Au NP hybrids are expected to be activated under nearinfrared light, which can penetrate more deeply into the body. ${ }^{88}$
Attempts have already been made to develop PEG-modified dendrimer-Au NP hybrids that can be activated with near-infrared light. ${ }^{89}$ 


\section{CONCLUSION}

Dendrimers with a regularly branched tree-like backbone and globular shape are unique molecules that differ from conventional linear polymers. Their highly defined 3D structure makes them very attractive for use as base materials for application to various fields, including biomedical fields. To increase their usefulness and to extend the range of their application, the functionalization of dendrimers is necessary. The surface modification, molecular assembly and hybrid formation described in this article are efficient approaches to the production of dendrimer-based nanomaterials with bio-related functions. Although these nanomaterials are presently still being improved, they will contribute to the creation of future medicines, such as those that are highly target-specific and non-invasive.

1 Tomalia, D. A., Baker, H., Dewald, J., Hall, M., Kallos, G., Martin, S., Roeck, J., Ryder, J. \& Smith, P. A. New class of polymers: starburst-dendritic macromolecules. Polym. J. 17, 117-132 (1985)

2 Tomalia, D. A., Naylor, A. M. \& Goddard, III W. A. Sturburst dendrimers: molecularlevel control of size, shape, surface chemistry, topology, and flexibility from atoms to macroscopic matter. Angew. Chem. Int. Ed. Engl. 29, 138-175 (1990).

3 Grayson, S. M. \& Frechet, J. M. J. Convergent dendrons and dendrimers: from synthesis to applications. Chem. Rev. 101, 3819-3867 (2001).

4 Newkome, G. R. \& Shreiner, C. D. Poly(amidoamine), polypropylenimine, and related dendrimers and dendrons possessing different $1-2$ branching motifs: an overview of the divergent procedures. Polymer 49, 1-173 (2008).

5 Lee, C. C., MacKay, J. A., Frechet, J. M. J. \& Szoka, F. C. Designing dendrimers for biological applications. Nat. Biotech. 23, 1517-151526 (2005).

6 Fischer, M. \& Vogtle, F. Dendrimers: from design to application-a progress report. Angew. Chem. Int. Ed. Engl. 38, 885-905 (1999).

7 Medina, S. H. \& El-Sayed, M. E. H. Dendrimers as carriers for delivery of chemotherapeutic agents. Chem. Rev. 109, 3141-3157 (2009).

8 Guillot-Nieckowski, M., Eisler, S. \& Diederich, F. Dendritic vectors for gene transfection. New J. Chem. 31, 1111-1127 (2007).

9 Malik, N., Wiwattanapatapee, R., Klopsh, R., Lorenz, K., Frey, H., Weener, J. W., Meijer, E. W., Paulus, W. \& Duncan, R. Dendrimers: relationship between structure and biocompatibility in vitro, and preliminary studies on the biodistribution of i-125labelled polyamidoaminedendrimersi in vivo. J. Control. Release 65, 133-148 (2000).

10 Duncan, R. The era of polymer therapeutics. Nat. Rev. Drug Delivery 2, 347-360 (2003).

11 Kojima, C., Kono, K., Maruyama, K. \& Takagishi, T. Synthesis of polyamidoaminedendrimers having polyethylene glycol grafts and their ability to encapsulate anticancer drugs. Bioconjugate Chem. 11, 910-917 (2000).

12 Twyman, L. J., Beezer, A. E., Esfand, R., Hardy, M. J. \& Mitchell, J. C. The synthesis of water soluble dendrimers, and their application as possible drug delivery systems.. Tetrahedron Lett. 40, 1743-1746 (1999).

13 Kojima, C., Toi, Y., Harada, A. \& Kono, K. Preparation of polyethylene glycol-attached dendrimers encapsulating photosensitizers for application to photodynamic therapy. Bioconjugate Chem. 18, 663-670 (2007).

14 Liu, M., Kono, K. \& Frechet, J. M. J. Water-soluble dendritic unimolecular micelles: their potential as drug delivery agents. J. Control. Release 65, 121-131 (2000).

15 Jansen, J. F. G. A., de Brabander-van den Berg, E. M. M. \& Meijer, E. W. Encapsulation of guest molecules into a dendritic box. Science 266, 1226-1229 (1994).

16 Kono, K., Fukui, T., Takagishi, T., Sakurai, S. \& Kojima, C. Preparation of poly(ethylene glycol)-modified poly(amidoamine) dendrimers with a shell of hydrophobic amino acid residues and their function as a nanocontainer. Polymer 49, 2832-2838 (2008).

17 Zimmerman, S. C., Wendland, M. S., Rakow, N. A Z Zharov, I. \& Suslick, K. S. Synthetic hosts by monomolecular imprinting inside dendrimers. Nature 418, 399403 (2002).

18 Wendland, M. S. \& Zimmerman, S. C. Synthesis of cored dendrimers. J. Am. Chem. Soc. 121, 1389-1390 (1999).

19 Schultz, L. G., Zhao, Y. \& Zimmerman, S. C. Synthesis of cored dendrimers with internal cross-links. Angew. Chem. Int. Ed. Engl. 40, 1962-1966 (2001).

20 Haba, Y., Harada, A., Takagishi, T. \& Kono, K. Synthesis of biocompatible dendrimers with a peripheral network formed by linking of polymerizable groups. Polymer 46, 1813-1820 (2005)

21 Kojima, C., Haba, Y., Fukui, T., Kono, K. \& Takagishi, T. Design of biocompatible dendrimers with environment-sensitivity. Macromolecules 36, 2183-2186 (2003).

22 Pistolis, G., Malliaris, A., Tsiourvas, D. \& Paleos, C.M. Poly(propyleneimine) dendrimers as ph-sensitive controlled-release systems. Chem. Eur. J. 5, 1440-1444 (1999).

23 Paleos, C.M., Tsiourvas, D., Sideratou, Z. \& Tziveleka, L. Acid- and salt-triggered multifunctional poly(propylene imine) dendrimer as a prospective drug delivery system. Biomacromolecules 5, 524-529 (2004).
24 Liu, Y., Bryantsev, V. S., Diallo, M. S. \& Goddard, III W. A. PAMAM dendrimers undergo $\mathrm{pH}$ responsive conformational changes without swelling. J. Am. Chem. Soc. 131, 2798-2799 (2009).

25 Archut, A., Azzellini, G. C., Balzani, V., DeCola, L. \& Vogtle, F. Toward photoswitchable dendritic hosts. Interaction between azobenzene-functionalized dendrimers and eosin. J. Am. Chem. Soc. 120, 12187-12191 (1998).

26 Li, Y., Jia, X., Gao, M., He, H., Kuang, G. \& Wei, Y. Photoresponsive nanocarriers based on PAMAM dendrimers with a o-nitrobenzyl shell. J. Polym. Sci. A Polym. Chem. 48, 551-557 (2010)

27 Nakayama, M. \& Okano, T. Multi-targeting cancer chemotherapy using temperatureresponsive drug carrier systems. React. Funct. Polym. 71, 235-244 (2011).

28 Chung, J. E., Yokoyama, M., Yamato, M., Aoyagi, T., Sakurai, Y. \& Okano, T. Thermoresponsive drug delivery from polymeric micelles constructed using block copolymers of poly(n-isopropylacrylamide) and poly(butylmethacrylate). J. Control. Release 62, 115-127 (1999).

29 Kono, K. Thermosensitive polymer-modified liposomes. Adv. Drug Delivery Rev. 53, 307-319 (2001).

30 Kono, K., Ozawa, T., Yoshida, T., Ozaki, F., Ishizaka, Y., Maruyama, K., Kojima, C., Harada, A. \& Aoshima, S. Highly temperature-sensitive liposomes based on a thermosensitive block copolymer for tumor-specific chemotherapy. Biomaterials $\mathbf{3 1}$, 7096-7105 (2010).

31 Meng, F., Zhong, Z. \& Feijen, J. Stimuli-responsive polymersomes for programmed drug delivery. Biomacromolecules 197-209 (2009).

32 Schmaljohann, D. Thermo- and pH-responsive polymers in drug delivery. Adv. Drug Delivery Rev. 58, 1655-1670 (2006).

33 Heskins, M. \& Guillet, J. E. Solution properties of (N-isopropylacrylamide). J. Macromol. Sci. Chem. A2, 1441-1455 (1968)

34 Feil, H., Bae, Y. H., Feijen, J. \& Kim, S. W. Effect of comonomerhydrophilicity and ionization on the lower critical solution temperature of n-isopropylacrylamide copolymers. Macromolecules 26, 2496-2500 (1993).

35 Hayashi, H., Kono, K. \& Takagishi, T. Temperature-sensitive liposomes using copolymers of n-isopropylacrylamide. Bioconjug. Chem. 10, 412-418 (1999).

36 Kimura, M., Kato, M., Muto, T., Hanabusa, K. \& Shirai, H. Temperature-sensitive dendritic hosts: synthesis, characterization, and control of catalytic activity. Macromolecules 33, 1117-1119 (2000).

37 You, Y. Z., Hong, C. Y., Pan, C. Y. \& Wang, P. H. Synthesis of a dendritic coreshell nanostructure with a temperature-sensitive shell. Adv. Mater. 16, 1953-1957 (2004).

38 Hui, H., Fan, X. D. \& Cao, Z. L. Thermo- and pH-sensitive dendrimer derivatives with a shell of poly(N,N-dimethylaminoethyl methacrylate) and study of their controlled drug release behavior. Polymer 46, 9514-9522 (2005).

39 Zhao, Y., Fan, X., Liu, D. \& Wang, Z. PEGylated thermo-sensitive poly(amidoamine) dendritic drug delivery systems. Int. J. Pharm. 409, 229-236 (2011).

40 Haba, Y., Harada, A., Takagishi, T. \& Kono, K. Rendering poly(amidoamine) or poly(propylenimine) dendrimers temperature sensitive. J. Am. Chem. Soc. 126, 12760-12761 (2004)

41 Suwa, K., Wada, Y., Kikunaga, Y., Morishita, K. \& Akashi, M. Synthesis and functionalities of poly( $\mathrm{N}$-vinylalkylamide). 4 . Synthesis and free radical polymerization of $\mathrm{N}$-vinylisobutyramide and thermosensitive properties of the polymer. J. Polym. Sci. A Polym. Chem. 35, 1763-1768 (1997)

42 Ito, S. Phase transition of aqueous solution of poly( $\mathrm{N}$-alkylacrylamide) derivatives: effects of side chain structure. Kobunshi Ronbunshu 46, 437-443 (1988).

43 Suwa, K., Morishita, K., Kishida, A. \& Akashi, M. Synthesis and functionalities of poly(N-vinylalkylamide). 5. Control of a lower critical solution temperature of poly (N-vinylalkylamide). J. Polym. Sci. A Polym. Chem. 35, 3087-3094 (1997).

44 Haba, Y., Kojima, C., Harada, A. \& Kono, K. Control of temperature-sensitive properties of poly(amidoamine) dendrimers using peripheral modification with various alkylamide groups. Macromolecules 39, 7451-7453 (2006)

45 Haba, Y., Kojima, C., Harada, A. \& Kono, K. Comparison of thermosensitive properties between poly(amidoamine) dendrimers having peripheral $\mathrm{N}$-isopropylamide groups and linear polymers with the same groups. Angew. Chem. Int. Ed. Engl. 46, 234-237 (2007).

46 Kono, K., Miyoshi, T., Haba, Y., Murakami, E., Kojima, C. \& Harada, A. Temperature sensitivity control of alkylamide-terminated poly(amidoamine) dendrimers induced by guest molecule binding. J. Am. Chem. Soc. 129, 7222-7223 (2007).

47 Tono, Y., Kojima, C., Haba, Y., Takahashi, T., Harada, A., Yagi, S. \& Kono, K. Thermosensitive properties of poly(amidoamine) dendrimers with peripheral phenylalanine residues. Langmuir 22, 4920-4922 (2006).

48 Parrott, M. C., Marchington, E. B., Valliant, J. F. \& Adronov, A. Synthesis and properties of carborane-functionalized aliphatic polyester dendrimers. J. Am. Chem. Soc. 127, 12081-12089 (2005)

49 Guo, Z., Zhang, Y., Huang, W., Zhou, Y. \& Yan, D. Terminal modification with 1 -adamantylamine to endow hyperbranched polyamidoamine with thermo-/pH-responsive properties. Macromol. Rapid Commun. 29, 1746-1751 (2008).

50 Liu, H., Chen, Y. \& Shen, Z. J. Thermoresponsive hyperbranchedpolyethylenimines with isobutyramide functional groups. Polym. Sci. A Polym. Chem. 45, 1177-1184 (2007).

51 Wang, H., Sun, S. \& Wu, P. Thermodynamics of hyperbranched poly(ethylenimine) with isobutyramide residues during phase transition: an insight into the molecular mechanism. J. Phys. Chem. B 115, 8832-8844 (2011).

52 Kojima, C., Yoshimura, K., Harada, A., Sakanishi, Y. \& Kono, K. Synthesis and characterization of hyperbranched poly(glycidol) modified with $\mathrm{pH}$ - and temperaturesensitive groups. Bioconjugate Chem. 20, 1054-1057 (2009). 
53 Kojima, C., Yoshimura, K., Harada, A., Sakanishi, Y. \& Kono, K. Temperature-sensitive hyperbranched poly(glycidol)s with oligo(ethylene glycol) monoethers. J. Polym. Sci. A Polym. Chem. 48, 4047-4054 (2010).

54 Sun, X., Zhou, Y. \& Yan, D. Rendering hyperbranched polyglycerol adjustably thermoresponsive by adamantyl modification and host/guest interaction. Macromol. Chem. Phys. 211, 1940-1946 (2010).

55 Shen, Y., Kuang, M., Shen, Z., Nieberle, J., Duan, H. \& Frey, H. Gold nanoparticles coated with a thermosensitive hyperbranched polyelectrolyte: towards smart temperature and $\mathrm{pH}$ nanosensors. Angew. Chem. Int. Ed. Engl. 47, 2227-2230 (2008).

$56 \mathrm{Jia}$, Z., Chen, H., Zhu, X. \& Yan, D. Backbone-thermoresponsive hyperbranched polyethers. J. Am. Chem. Soc. 128, 8144-8145 (2006).

57 Chen, H., Jia, Z., Yan, D. \& Zhu, X. Thermo-responsive highly branched polyethers by proton-transfer polymerization of 1,2,7,8-diepoxyoctane and multiols. Macromol. Chem. Phys. 208, 1637-1645 (2007)

58 Israelachvili, J. N., Marcelja, S. \& Horn, R. G. Physical principles of membrane organization. Q. Rev. Biophys 13, 121-200 (1980).

59 Rosen, B. M. Wilson, C. J., Wilson, D. A., Peterca, M., Imam, M. R. \& Percec, V. Dendron-mediated self-assembly, disassembly, and self-organization of complex systems. Chem. Rev. 109, 6275-6540 (2009).

60 Tsuda, K., Dol, G. C., Gensch, T., Hofkens, J., Latterini, L., Weener, J. W., Meijer, E. W. \& De Schryver, F. C. Fluorescence from azobenzene functionalized poly(propylene imine) dendrimers in self-assembled supramolecular structures. J. Am. Chem. Soc. 122, 3445-3452 (2000)

61 Schenning, A. P. H. J., Elissen-Roman, C., Weener, J. W., Baars, M. W. P. L., van der Gaast, S. J. \& Meijer, E. W. Amphiphilic dendrimers as building blocks in supramolecular assemblies. J. Am. Chem. Soc. 120, 8199-8208 (1998).

62 van Hest, J. C. M., Delnoye, D. A. P., Baar, M. W. P. L., van Genderen, M. H. P. \& Meijer, E. W. Polystyrene-dendrimer amphiphilic block-copolymers with a generationdependent aggregation. Science 268, 1592-1595 (1995).

63 Harada, A., Nakanishi, K., Ichimura, S., Kojima, C. \& Kono, K. Spontaneous formation of narrowly-distributed self-assembly from polyamidoamine dendron-poly(L-lysine) block copolymers through helix-coil transition of poly(L-lysine) block. J. Polym. Sci. A Polym. Chem. 47, 1217-1223 (2009).

64 Takahashi, T., Kono, K., Itoh, T., Emi, N. \& Takagishi, T. Synthesis of novel cationic lipids having polyamidoaminedendrons and their transfection activity. Bioconjugate Chem. 14, 764-773 (2003).

65 Takahashi, T., Kojima, C., Harada, A. \& Kono, K. Alkyl chain moieties of polyamidoamine dendron-bearing lipids influence their function as a nonviral gene vector. Bioconjugate Chem. 18, 1349-1354 (2007).

66 Takahashi, T., Hirose, J., Kojima, C., Harada, A. \& Kono, K. Synthesis of poly(amidoamine) dendron-bearing lipids with poly(ethylene glycol) grafts and their use for stabilization of nonviral gene vectors. Bioconjugate Chem. 18, 1163-1169 (2007).

67 Takahashi, T., Yuba, E., Kojima, C., Harada, A. \& Kono, K. Synthesis of a polyamidoamine dendron-bearing lipid having sugar moieties and its use for preparation of nonviral gene vectors. Res. Chem. Intermediat. 35, 1005-1014 (2009).

68 Kono, K., Murakami, E., Hiranaka, Y., Yuba, E., Kojima, C., Harada, A. \& Sakurai, K. Thermosensitive molecular assemblies from polyamidoamine dendron-based lipids. Angew. Chem. Int. Ed. Engl. 50, 6332-6336 (2011).

69 Yatvin, M. B., Weinstein, N. J. N., Dennis, W. H. \& Blumenthal, R. Design of liposomes for enhanced local release of drugs by hyperthermia. Science 202, 1290-1292 (1978).

70 Astruc, D., Boisselier, E. \& Ornelas, C. Dendrimers designed for functions: from physical, photophysical, and supramolecular properties to applications in sensing, catalysis, molecular electronics, photonics, and nanomedicine. Chem. Rev. 110, 1857-1959 (2010)

71 Bronstein, L. M. \& Shifrina, Z. B. Dendrimers as encapsulating, stabilizing, or directing agents for inorganic nanoparticles. Chem. Rev. 111, 5301-5344 (2011).

72 Crooks, R. M., Zhao, M., Sun, L., Chechik, V. \& Yeung, L. K. Dendrimer-encapsulated metal nanoparticles: synthesis, characterization, and applications to catalysis. Acc. Chem. Res. 34, 181-190 (2001).

73 Twyman, L. J., King, A. S. H. \& Martin, I. K. Catalysis inside dendrimers. Chem. Soc Rev. 31, 69-82 (2002).

74 Zheng, J., Zhang, C. \& Dickson, R. M. Highly fluorescent, water-soluble, size-tunable gold quantum dots. Phys Rev. Lett. 93, 077402/1-077402/4 (2004).

75 Krasteva, N., Besnard, I., Guse, B., Bauer, R. E., Mullen, K., Yasuda, A. \& Vossmeyer, T. Self-assembled gold nanoparticle/dendrimer composite films for vapor sensing applications. Nano. Lett. 2, 551-555 (2002).

76 Guo, R., Wang, H., Peng, C., Shen, M., Pan, M., Cao, X., Zhang, G. \& Shi, X. X-Ray attenuation property of dendrimer-entrapped gold nanoparticles. J. Phys. Chem. C 114 50-56 (2010).

77 Kojima, C., Umeda, Y., Ogawa, M., Harada, A., Magata, Y. \& Kono, K. X-Ray computed tomography contrast agents prepared by seeded growth of gold nanoparticle in pegylateddendrimer. Nanotechnology 21, 245104 (2010).

78 Shen, M. \& Shi, X. Dendrimer-based organic/inorganic hybrid nanoparticles in biomedical applications. Nanoscale 2, 1596-1610 (2010).

79 Shi, X., Wang, S., Meshinchi, S., Van Antwerp, M.E., Bi, X., Lee, I. \& Baker, Jr. J.B. Dendrimer-entrapped gold nanoparticles as a platform for cancer-cell targeting and imaging. Small 3, 1245-1252 (2007).

80 Daniel, M. C. \& Astruc, D. Gold nanoparticles: assembly, supramolecular chemistry, quantum-size-related properties, and applications toward biology, catalysis, and nanotechnology. Chem. Rev. 104, 293-346 (2004).

81 Sonvico, F., Dubernet, C., Colombo, P. \& Couvreur, P. Metallic colloid nanotechnology, applications in diagnosis and therapeutics. Curr. Pharm. Des. 11, 2091-2105 (2005).

$82 \mathrm{Hu}$, M., Chen, J., Li, Z. Y., Au, L., Hartland, G. V., Li, X., Marquez, M. \& Xia, Y. Gold nanostructures: engineering their plasmonic properties for biomedical applications. Chem. Soc. Rev. 35, 1084-1094 (2006).

83 Govorov, A. O. \& Richardson, H. H. Generating heat with metal nanoparticles. Nano Today 2, 30-38 (2007).

84 Grohn, F., Bauer, B. J., Akpalu, Y. A., Jackson, E. J. \& Amis, E. Dendrimer templates for the formation of gold nanoclusters. Macromolecules 33, 6042-6050 (2000).

85 Hedden, R. C., Bauer, B. J., Smith, A. P., Grohn, F. \& Amis, E. Templating of inorganic nanoparticles by PAMAM/PEG dendrimer-star polymers. Polymer 43, 5473-5481 (2002)

86 Haba, Y., Kojima, C., Harada, A., Ura, T., Horinaka, H. \& Kono, K. Preparation of poly(ethylene glycol)-modified poly(amidoamine) dendrimers encapsulating gold nanoparticles and their heat-generating ability. Langmuir 23, 5243-5246 (2007).

87 Umeda, Y., Kojima, C., Harada, A., Horinaka, H. \& Kono, K. PEG-attached PAMAM dendrimers encapsulating gold nanoparticles: growing gold nanoparticles in the dendrimers for improvement of their photothermal properties. Bioconjugate Chem. 21, 1559-1564 (2010).

88 Weissleder, R. A. Clearer vision for in vivo imaging. Nat. Biotech. 19, 316-317 (2001).

89 Kojima, C., Umeda, Y., Harada, A. \& Kono, K. Preparation of near-infrared light absorbing gold nanoparticles using polyethylene glycol-attached dendrimers. Colloids Surf. B: Biointerfaces 81, 648-651 (2010).

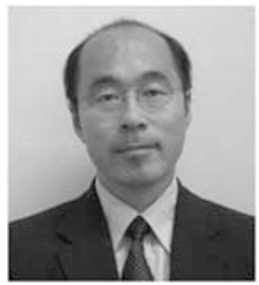

Kenji Kono was born in Kanagawa prefecture in 1959, and graduated from the Department of Polymer Chemistry of Kyoto University in 1989. He received his PhD degree from Graduate School of Engineering, Kyoto University, Japan in 1990. He worked as a Research Associate of Osaka Prefecture University, Japan, from 1989 to 1994, as an Associate Professor from 1994 to 2002 and as a Professor from 2002 to date in Graduate School of Engineering, Osaka Prefecture University. He also served as a visiting scholar at University of California, Berkeley from 1997 to 1998. His research interests are polymer-based nanomaterials with bio-related functions for application to biomedical fields, including drug delivery and nanomedicines. 